\title{
Adaptive streaming media VOD system under the background of new media
}

\author{
Yunxiang Zhang ${ }^{1, \mathrm{a}}$,Yunpeng $\mathrm{He}^{2, \mathrm{~b}}$, Shengcan $\mathrm{Jin}^{3, \mathrm{c}}$ and Bin Wang ${ }^{4, \mathrm{~d}_{*}}$ \\ ${ }^{1}$ Business College Jiamusi University, Jiamusi China \\ ${ }^{2}$ Radio and Television Bureau of Jiamusi , iamusi China \\ ${ }^{3}$ Business College Jiamusi University, Jiamusi China \\ ${ }^{4}$ College of Information Science \& Electronic Technology,Jiamusi University,Jiamusi China \\ ajmsuzyx@163.com, ${ }^{\mathrm{b}} 18634662 @ q q . c o m,{ }^{c}$ jinsc@163.com, ${ }^{\mathrm{d}} \mathrm{jm}$ suwang@163.com
}

Keywords: VOD; streaming media; Adaptive

\begin{abstract}
In this thesis, the main part is the research on the Video-On-Demand. Using an adaptive streaming media transmission program, according to network conditions on the video-on-demand server control pro tournament at the same time adjust the client's cache, so that the client be able to smoothly carry out high-quality media player. The paper puts forward the overall design scheme of streaming media adaptive transmission system, the scheme covers rate shaping, congestion control and video quality adaptation function modules and their mutual relations, each module can be applied demand selection algorithm is realized, which improves the flexibility and scalability of the scheme. So the scheme doesn't depend on specific video encoding standards and network protocols, wide range of application.
\end{abstract}

\section{Introduction}

In recent years, with the rapid development of science and technology, new media has attracted more and more attention, become a hot topic with eristic people. The prosperity of new media in the industry also makes scholars to further strengthen its research, new media is relative to the traditional media, new media form the traditional media newspapers, radio, television and other later developed, is the use of digital technology, network technology, mobile technology, through the Internet, wireless communication network, cable network etc. channel and computer, mobile phone, digital TV terminal, providing communication form and the form of media information and entertainment to users. Requirement for people to enjoy a better video services via the Internet is becoming more and more urgent. In order to meet the needs of users, people must adopt new methods to realize the transmission and video streaming media technology, streaming media technology to save users time, improves the utilization rate of resources, therefore, is the development of streaming media technology to promote application of video on demand.

In the new media context, the core problem of restricting the development of stream media broadband application service quality. Say simply, because the Internet is not reliable, its bandwidth, load changes acutely, it is difficult to meet the real-time stream media service quality of broadband service requirements, and often play jam, delay, jitter severe video quality bad situation, cause very big effect to the user on the senses; promotion also hindered the streaming media technology. At present, more and more people pay attention to is the study of VOD system based on streaming media technology and stream media adaptive transmission technology, so the combination of the two can't solve the problem? Therefore, this paper proposes a new adaptive streaming media system based on VOD, it is mainly to certain measures on both the server and the client terminal, if can get a satisfactory effect can demand at low network bandwidth, will further stimulate the people using the VOD system's enthusiasm. 


\section{The basic idea of system}

(1) in the server using the network congestion control of some of the sending rate control algorithm for video data. Doing so can achieve 3 Effects: congestion control algorithm can detect the available network bandwidth, server can change the reference available network bandwidth in the process of work.;Transmitted at a rate of media data is not greater than the available network bandwidth, network packet loss rate will be relatively small. 3) congestion control algorithm to make the media business flow with the TCP in the network business flow of fair competition on the network bandwidth.

(2) were observed in the client buffer, and in the process of sending video data in trying to control the buffer data volume in a certain range. If the amount of data the client buffer has been effectively controlled in a certain range, so you can ensure that the client playback is continuous and timely, because there is always the video data and audio data buffer; in addition, can also be considered to influence of network delay on the media data transmission process is absorbed by buffer.

(3) according to the video quality control algorithm for streaming video quality in real-time adjustment, in order to achieve the same bit rate video stream network bandwidth matching. Theoretical analysis and simulation results have shown that the effect of control algorithm of video quality.

So, the congestion control, video quality control and rate shaping can be the network packet loss rate control in a relatively low level, the transmission of video data and process with changing network bandwidth matching, and offset the impact of network delay fluctuation on the video data transmission process. So, speaking from the principle of client playback quality can be guaranteed.

\section{The system design scheme}

Scheme design is shown in Figure 1, the dotted line on the left is the hard disk I/O control module server to achieve. The whole transmission scheme consists of 5 functional modules of the dotted line on the right side of the. Control project with the video stream as the control object, the audio stream rate is generally small, so the sending process without extra control, so only the synchronized with video.

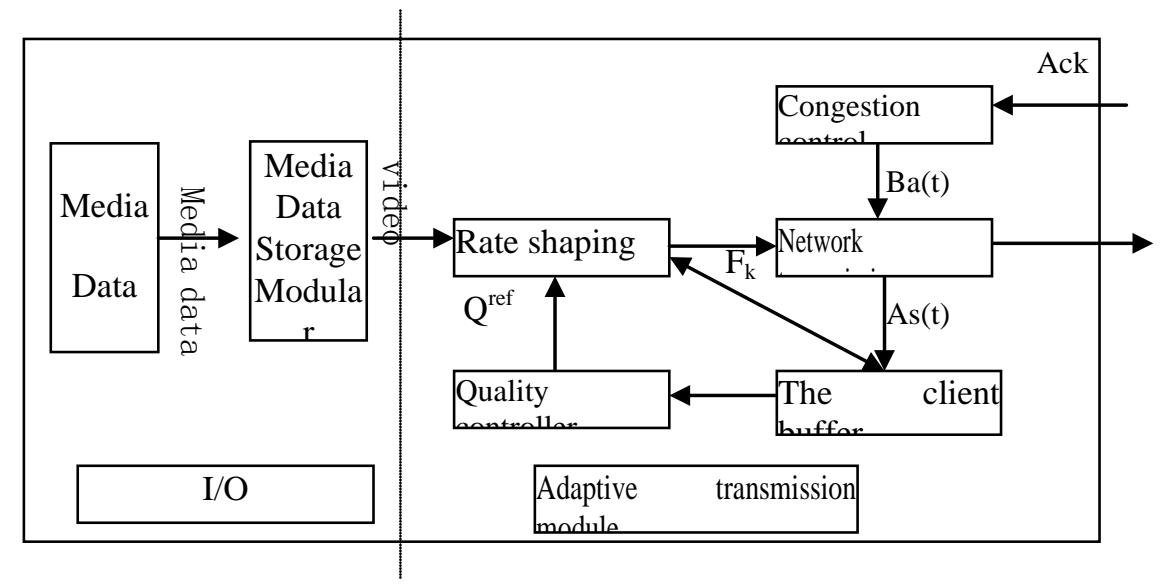

Fig. 1 transmission scheme of adaptive module

(1) congestion control: the main function is according to the network packet loss rate of client feedback back information congestion control, bandwidth available to Ba(t) to detect network.

We need to RTCP packet processing to obtain the packet loss rate, followed by the need for packet loss rate by using the reasonable strategy for congestion control.

In this paper, first in the Clear Server in the design and implementation of a RTCP module, for RTCP communication between server and client maintenance. Because the RTCP function have been realized by the client player, so as long as the RTCP module Clear Server in accordance with the RTCP communication between the server and the client protocol specification can smoothly. 
RTCP module Clear Server in the specified RTCP data network port receiving client feedback packets, and analyzing and processing, between server and client both audio and visual communication by data packet loss rate, network delay jitter and other information.

And the implementation of specific process design RTCP module involves comparing the bottom in the software design part, here is not a repeat.

Congestion control is the core part of congestion control algorithms, namely the change trend of how to detect the network bandwidth according to the change of network packet loss rate. Since the feedback cycle of RTCP data packet is relatively long, generally for 5 seconds, so can not directly use some TCP friendly congestion control algorithm of congestion control algorithm, and the need to design a new realization. Used in this paper is the fourth chapter improved AIMD congestion control algorithm.

(2) the network to send: according to $\mathrm{Ba}(\mathrm{t})$ video frames will rate shaping module for processing network sent packing, and ensure the transmission rate of the video data is not more than $\mathrm{Ba}(\mathrm{t})$. Ba $(\mathrm{t})$ defines a video data transmitting rate, so the loss of the video data rate can be controlled.

(3) the client buffer: according to the sending rate of actual network As network transmission module $(\mathrm{T})$ and rate shaper rate shaping after each video frame size Fk, recursive estimate the client buffer data size in $\mathrm{Br}(\mathrm{t})$. A recursive formula for the

(1)

$$
\operatorname{Br}(\mathrm{t})=\operatorname{Br}(\mathrm{t}-1)+\operatorname{As}(\mathrm{t}) \quad-\quad \mathrm{Fk}
$$

Its specific meaning is: the client buffer data volume is equal to the video frame on a time data minus the amount just play the size, the amount of video data plus send the time server.

(4) quality controller: according to $\mathrm{Br}(\mathrm{T})$ and its set value difference of $\mathrm{Br}$ between ref, using the PID control algorithm to obtain the video quality reference current value Qref.

The specific formula for PID control algorithm

$$
\left\{\begin{array}{l}
\mathrm{I}(\mathrm{t})=\mathrm{I}(\mathrm{t}-1)+\mathrm{K}_{\mathrm{i}} \times\left[\mathrm{Br}(\mathrm{t})-\mathrm{Br}^{\text {ref }}\right] \\
\mathrm{Q}^{\text {ref }}=\mathrm{K}_{\mathrm{p}} \times\left[\mathrm{Br}(\mathrm{t})-\mathrm{Br}^{\text {ref }}\right]+\mathrm{I}(\mathrm{t})
\end{array}\right.
$$

Where $K_{i}$ and $K_{p}$ are constant and proportional integral. The goal of the controller is regulated by Qref, the client buffer data quantity control near its set value.

(5) rate shaper: rate shaper based on Qref for rate shaping the video stream, by adjusting the video quality video flow rate adjustment to Qref times the original rate. All modules work together will be able to send process, send rate and video streaming video stream itself rate control, the fluctuation of the network environment, guarantee the smooth video data, timely to client.

\section{System simulation}

Clear Serve in the application of the seeding quality adaptive transmission scheme in the case of. In this case, not only the video data sending rate with the change of the network bandwidth and the corresponding adjustment, video streaming video quality also made adjustments to make, bit rate video stream with the network bandwidth matching, to ensure smooth playback of the client. On demand effects such as Figure 2 to figure 4. In Figure 2, 100ms cycle sampling, network bandwidth and transmission rate of the unit is bits/s. As can be seen, the congestion control module basically followed the changes of the network bandwidth and transmission rate; the server is not larger than the congestion control module to get the network bandwidth. At the same time, the video quality is low, the server sending rate may be less than the congestion control module to get the network bandwidth. Figure 3 is the variation curves of reference video quality. Visible video reference quality fluctuates between 0.4 to 0.7 , the curve is relatively smooth. By the values of the above the output of the Brref controller has a great influence on the quality, will directly affect the control effect. Especially for the duration of a few minutes of the movie file, its rate characteristic may with the scene change change a lot, that in different period the average bit rate have great difference. 
Now if only according to the average bit rate of the video stream total set Brref words appears a little stiff, the control effect will be affected to a certain extent. Therefore, the best way is adaptive transmission scheme in the running process, according to the change of Brref value greatly adjust the video stream rate, ensure that the average bit rate in each time its value with this time matching. Under this strategy adjusts to the bit rate video stream with the available bandwidth is reduced to match the level of video quality. In this way, on one hand we have video bit rate is reduced to the range of available network bandwidth allowed, on the other hand will therefore video quality caused by fluctuation is reduced to the minimum, ensure that the client enjoy more stable video playback. Figure 4 is the data variation curve of the client buffer. Visible $\mathrm{Br}(\mathrm{t})$ has been around Brref fluctuations, although more violent fluctuation. But always in the range of validity is the client buffer has been no overflow and underflow condition.

Frame dropping strategy for rate shaping in the video stream, for a single video frame can determine whether to drop, so its accuracy is relatively low. Moreover, because the Clear Server transmission cycle is short, only 300ms, the number of video frames so frame dropping strategy can handle only every time 7 to 10 (with a frame rate of 25 frames per second for example). In this case, because the lost frame rate shaping errors caused by the more. As shown in Figure 5, the curve in the figure is at a time when the error rate shaping. Error is defined as: Error= (the actual amount of data needs to be discarded discarded a amount of data) / need to discard data quantity. Among them, the amount of data need to discard rate shaping module is calculated according to the total data amount of the current video frame and the reference video quality. As can be seen from the graph, the error is concentrated in the interval -0.2 to 0.2 , sometimes can reach 0.4 or even 0.6. So, basically every rate shaping errors will occur, as a control system, actuator adaptive transmission schemes in the presence of errors, it will certainly have an impact on the control effect. If the use of layered coding or fine granularity coding application server in the video stream, or it can be applied to some typical DAS algorithm, in order to improve the accuracy rate shaper, believe that the adaptive transmission scheme control effect will be better.

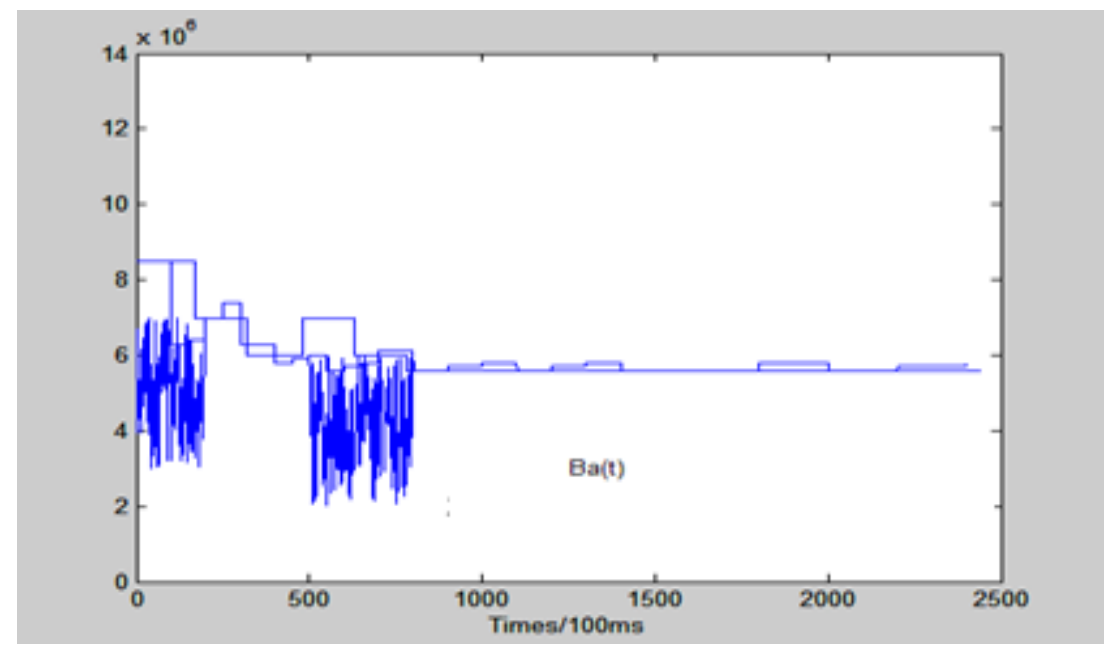

Fig. 2 The sending rate with the network bandwidth curve 


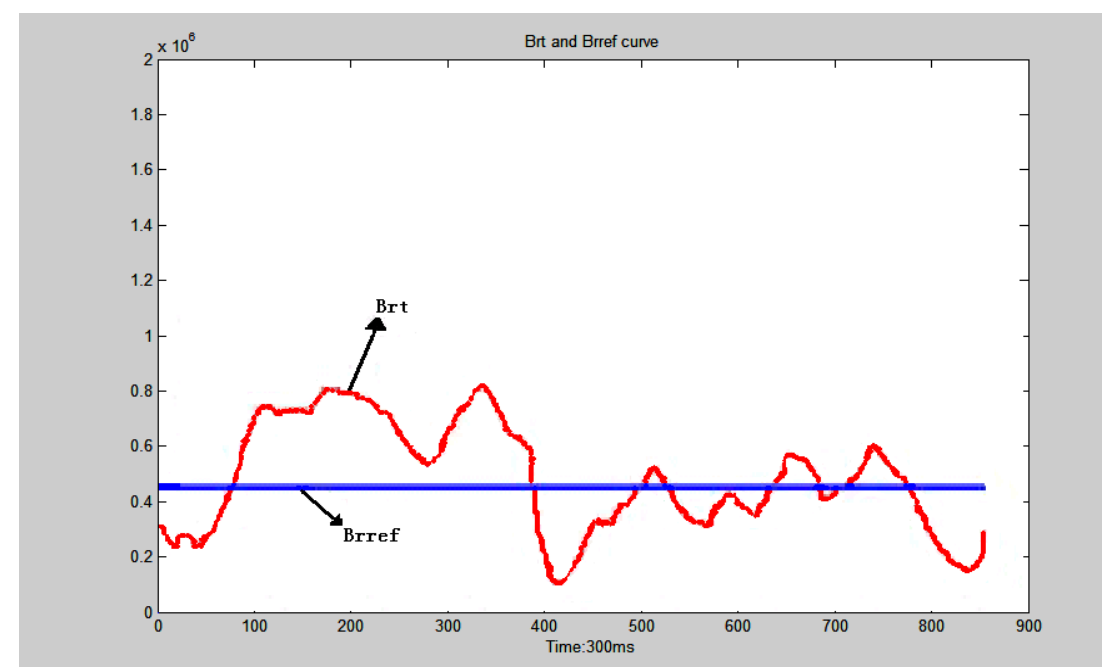

Figure 3 Reference video quality curve

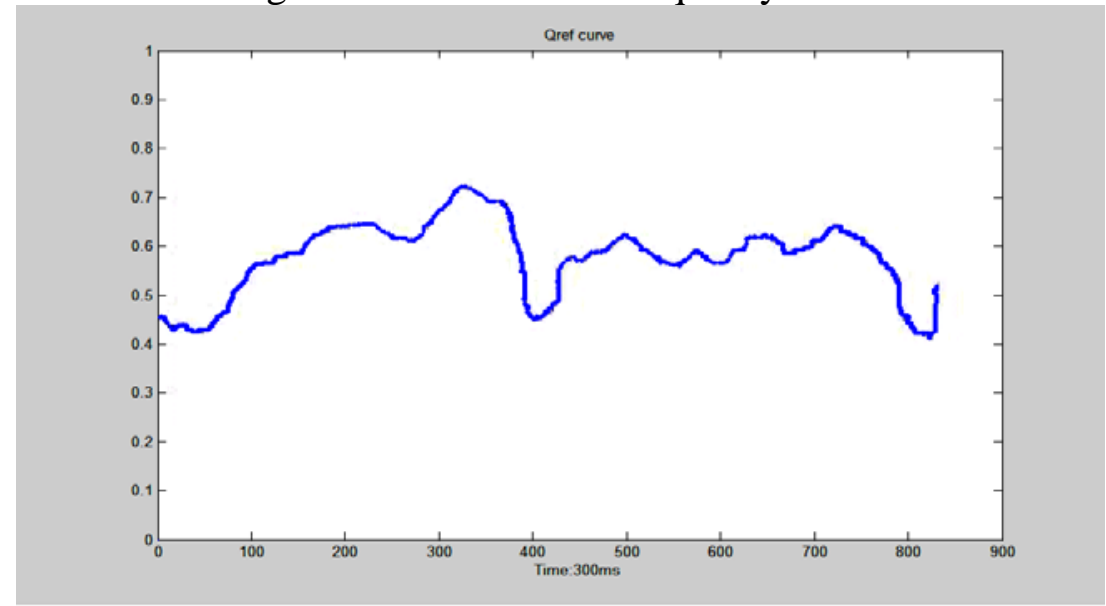

Fig. 4 The client buffer curve

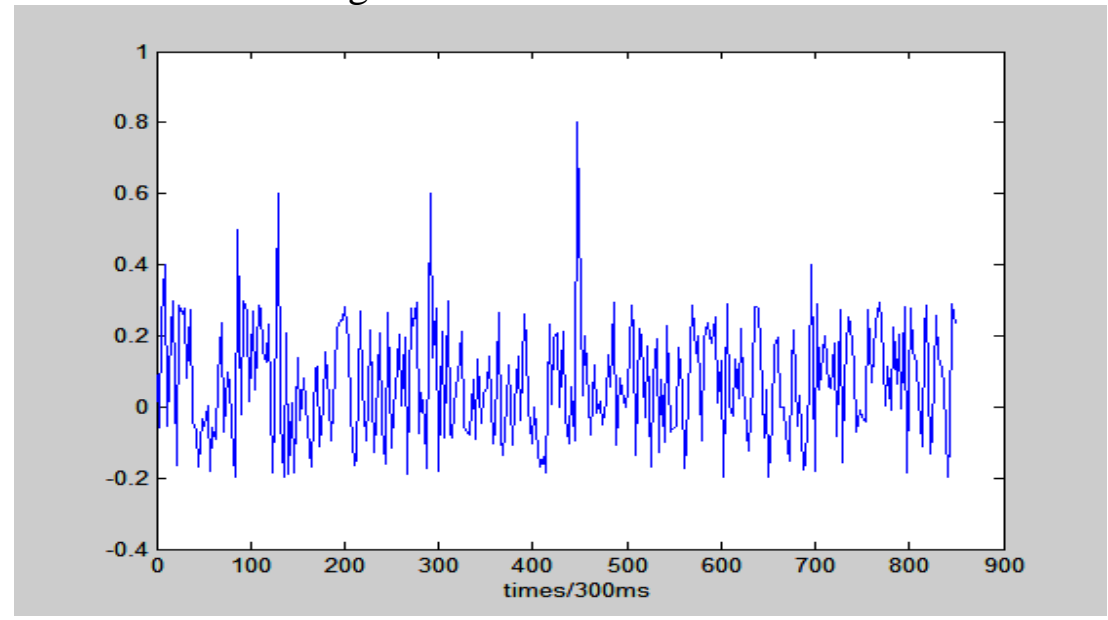

Fig. 5 Error curve of frame dropping strategy

\section{Conclusion}

From the chart we can see that: (1) congestion control module can detect network available bandwidth; (2) the amount of data the client buffer is controlled around the set value, although the more radical changes, but it is always meaningful, no underflow; (3) the change of quality reference value comparison gentle. At the same time, the client of the actual player is: (1) process the client playback is smooth, no pause phenomenon play; (2) the client playback of the video frame rate change, but the overall video quality changes slowly.

Adaptive streaming scheme has certain effect, in the network bandwidth fluctuation and under the condition of insufficient guarantee client enjoy better video. 


\section{Acknowledgement}

This research was supported by the National Science Foundation of Jiamusi University(Grant No . Lz2014-005) all support is gratefully acknowledged.

\section{References}

[1] H. Schulzrinne,S.Casner,R.Frederick, and V.Jacobson.RTP. A Transport Protocol for Real-Time Applications.RFC 1889,1996

[2] http://blog.csdn.net/da51e/archive/2004/12/10/211970.aspx[DB/OL]2006.12

[3] Dapeng Wu, Yiwei Thomas Hou .Transporting Real-Time Video overthelnternet: Challenges and Approaches. PROCEEDINGS OF THE IEEE, December 2000, 88(12): 1855-1874

[4] Cai N, Chiu D. Transport of MPEG-2 Video in Routed IP Networks: Effects of Network Congestion on Transport Stream Errors. France IDMS'99 Conf, October 1999

[5] http://www.vovida.org/protocols/downloads/rtp/[DB/OL],2006.10

[6] Zhengming Li, Zuo Zhang. Adaptive research on streaming media transmission scheme. Computer engineering and its application, 2006. 6: 226 228

[7] Hui Zhang, Chengliang Wang. Research on adaptive video streaming technology. Computer engineering and design, 2004, 2: 226 229

[8] http://www.live555.com [DB/OL], 2006.10

[9] http://research.edm.luc.ac.be/jori/jrtplib/documentation/index.html[DB/OL], 2006.10 